\title{
Bell's Expression and the Generalized Garg Form for Forced Dissociation of a Biomolecular Complex
}

\author{
Han-Jou Lin, ${ }^{1}$ Hsuan-Yi Chen, ${ }^{2, *}$ Yu-Jane Sheng, ${ }^{1, *}$ and Heng-Kwong Tsao ${ }^{3, *}$ \\ ${ }^{1}$ Department of Chemical Engineering, National Taiwan University, Taipei, Taiwan 106, Republic of China \\ ${ }^{2}$ Department of Physics, Graduate Institute of Biophysics, Center for Complex Systems, National Central University, \\ Jhongli, Taiwan 320, Republic of China \\ ${ }^{3}$ Department of Chemical and Materials Engineering, National Central University, Jhongli, Taiwan 320, Republic of China
}

(Received 27 June 2006; published 23 February 2007)

\begin{abstract}
The dissociation of a biomolecular complex under the action of constant force, constant loading rate, and periodic force is studied theoretically. We show that the celebrated Bell expression provides a good approximation for the bond dissociation rate when $F / F_{c} \ll 1$, where $F_{c}$ is the maxima slope of the binding potential along the reaction coordinate. When $1-F / F_{c} \ll 1$ the dissociation rate is better described by a generalized Garg form in which the potential derivative is expanded near $F_{c}$. We also show that a constant-force experiment is suitable for extracting the activation energy of the bond, a constant loading experiment is suitable to extract $F_{c}$, while time-periodic force can be applied to extract both bond dissociation rates at zero force and $F_{c}$.
\end{abstract}

DOI: 10.1103/PhysRevLett.98.088304

The dynamic strength of noncovalent biomolecular bonds has been experimentally explored by singlemolecule pulling experiments $[1,2]$. In these constant loading rate experiments, the strength of the bond is characterized by the relation between the rupture force $F_{u}$ and loading rate $\dot{F}_{t}$, i.e., the dynamic force spectroscopy. The main issue is how single-molecule pulling experiments should be analyzed to extract useful information associated with the kinetics and energy landscape along the dissociation pathway $[3,4]$.

The theoretical problem of finding the rupture force of a bond under constant loading rate corresponds to evaluating the escape time of a particle trapped in a one-dimensional potential well in the presence of both a time-ramped external force and thermal fluctuations, an interesting extension of the celebrated Kramers rate theory [5,6]. The primary role of external pulling in the dissociation kinetics is lowering the energy barrier $E_{a}$. Following the seminal work of Bell [7], it is often assumed that the metastable potential well is deep and the force-dependent energy barrier is $E_{a}(F)=E_{a}^{0}-F a$, where $E_{a}^{0}$ represents the intrinsic activation energy and $a$ the distance between the potential well and the barrier along the reaction coordinate. Up to now, Bell's expression is regarded as a phenomenological theory which is convenient to extract the intrinsic dissociation rate constant $k_{0}$ and $a$ from pulling experiments $[3,4]$. However, another work by Garg [8] assumed that bond rupture occurs when the metastable well becomes very shallow; in this case a cubic function is appropriate to approximate the potential surface in the vicinity of the critical force $\left(F_{c}\right)$ and $E_{a}(F)=E_{a}^{0}\left(1-F / F_{c}\right)^{3 / 2}$. Bell's expression yields $F_{u} \sim \ln \dot{F}_{t}$, while the Garg form results in $F_{u} \sim\left(\ln \dot{F}_{t}\right)^{2 / 3}$.

Recently a unified form with a fitting parameter $\nu$ [4] is proposed to extract $k_{0}, a$, and $E_{a}^{0}$. In this approach the escape rate under force $F$ is $k(F, \nu)=\frac{1}{\tau(F, \nu)} \times$
PACS numbers: 82.37.Np, 87.15.- $-v$

$\exp \left[-\beta E_{a}(F, \nu)\right]$, where the activation energy is $E_{a}(F, \nu)=E_{a}^{0}\left(1-\nu F a / E_{a}^{0}\right)^{1 / \nu}, \quad$ and $\quad \frac{1}{\tau(F, \nu)}=k_{0}(1-$ $\left.\nu F a / E_{a}^{0}\right)^{1 / \nu-1} e^{\beta E_{a}^{0}}$. Bell's expression is recovered for $\nu=$ 1 or $E_{a}^{0} \rightarrow \infty$, while the Garg form is obtained for $\nu=2 / 3$. It was suggested that this form is more general than Bell's expression. On the other hand, it has also been pointed out $[3,9]$ that for a single-well potential, Bell's expression and the Garg form are actually valid in different regimes. They correspond to two asymptotic limits: (i) the slow pulling regime $\left(F_{u} / F_{c} \ll 1\right)$ and (ii) the fast pulling regime (1 $F_{u} / F_{c} \ll 1$ ), respectively. Reference [3] further confirmed their claim by fitting atomic force microscope and biomembrane force probe measurement for unbinding biotinstreptavidin complex [2] to both limits.

To settle this issue and to provide a theoretical foundation for interpreting experimental data, in this Letter we derive Bell's expression and the generalized Garg form from Kramers rate theory and show that both of them are asymptotic expressions. The former is valid for weak pulling $\left(F / F_{c} \ll 1\right)$, and the latter is valid for strong pulling $\left(1-F / F_{c} \ll 1\right)$. We also discuss the bond lifetime in constant force, constant loading rate, and periodic force experiments. Our study shows that useful information about the bond can be extracted by performing different types of pulling experiment.

Barrier crossing under external force.-Consider an overdamped Brownian particle escaping from a metastable well of $U(x)$ under the action of force $F$, where $x$ is the reaction coordinate. The dynamics of this particle is described by the Langevin equation $M \gamma \frac{d x}{d t}=$ $-\frac{d}{d x}[U(x)-F x]+\xi(t)$. The friction coefficient $M \gamma$ is related to the diffusivity $D$ by $M \gamma=k_{B} T / D . M \gamma$ comes from the dissipation of kinetic energy into internal degrees of freedom. Therefore, $M \gamma$ in the trapped state may be significantly greater than that in the escaped state; in this case rebinding events can be neglected. 
Thermal force $\xi$ has zero mean and $\left\langle\xi(t) \xi\left(t^{\prime}\right)\right\rangle=$ $2 M \gamma k_{B} T \delta\left(t-t^{\prime}\right)$.

The free energy landscape associated with $U(x)$ is characterized by three parameters: the interaction range $a=$ $x_{+}^{0}-x_{-}^{0}$ which denotes the distance from the metastable well $x_{-}^{0}$ to the barrier $x_{+}^{0}$, the intrinsic energy barrier $E_{a}^{0}=$ $U\left(x_{+}^{0}\right)-U\left(x_{-}^{0}\right)$, and the maximum slope of the trapping potential, i.e., the critical force, $F_{c}=U^{\prime}\left(x_{c}\right)$. Typically $E_{a}^{0} \sim \mathcal{O}\left(10 k_{B} T\right), \quad a \sim \mathcal{O}(1 \mathrm{~nm}), \quad$ and $\quad F_{c} \sim \mathcal{O}(10 \mathrm{pN})$. When $F=0$, the biomolecular complex dissociation occurs due to thermal fluctuations. For $E_{a} \gg k_{B} T$, the escape rate is given by the Kramers theory $[5,6]$ :

$$
k=\tau^{-1} \exp \left(-\beta E_{a}\right), \quad \text { with } \quad \tau=\frac{2 \pi \gamma}{\omega_{-} \omega_{+}} .
$$

Here $\omega_{ \pm}=\left[\mp U^{\prime \prime}\left(x_{ \pm}\right) / M\right]^{1 / 2}$. This thermally activated escape denotes the kinetic limit. On the contrary, when $F>$ $F_{c}$ the binding force can be overcome without any help of thermal fluctuations. The dissociation is dominated by mechanical pulling; this is the mechanical limit. When $0<$ $F<F_{c}$, the external force alters the effective trapping potential to $V(x)=U(x)-F x$. Thus, the well and saddle positions are determined by $U^{\prime}\left(x_{ \pm}\right)=F$. The barrier height becomes $E_{a}(F)=V\left[x_{+}(F)\right]-V\left[x_{-}(F)\right], \tau$ becomes $\tau(F)$, and the rate constant at $F=0$ is $k_{0}=$ $\tau_{0}^{-1} e^{-\beta E_{a}^{0}}$. Because the energy barrier is lowered, the bond lifetime is reduced with increasing $F$.

Simple analytical expressions for the bond lifetime under constant force can be obtained in the asymptotic limits $F / F_{c} \ll 1$ and $1-F / F_{c} \ll 1$. When $F / F_{c} \ll 1$, $x_{ \pm}$is close to $x_{ \pm}^{0}$, and the trapping potential near $x_{ \pm}^{0}$ can be approximated by $U(x) \cong U\left(x_{ \pm}^{0}\right)+\frac{1}{2} U^{\prime \prime}\left(x_{ \pm}^{0}\right)\left(x-x_{ \pm}^{0}\right)^{2}$ since $U^{\prime}\left(x_{ \pm}^{0}\right)=0$. Therefore the new positions of well and saddle point are $x_{ \pm}(F) \cong x_{ \pm}^{0}+\frac{F}{U^{\prime \prime}\left(x_{ \pm}^{0}\right)}$. The forcedependent barrier height is then given by

$$
\begin{aligned}
E_{a}(F) & =V\left(x_{+}\right)-V\left(x_{-}\right) \\
& =E_{a}^{0}-F a+\frac{F^{2}}{2}\left[\frac{1}{U^{\prime \prime}\left(x_{-}^{0}\right)}-\frac{1}{U^{\prime \prime}\left(x_{+}^{0}\right)}\right]
\end{aligned}
$$

and $U^{\prime \prime}\left(x_{ \pm}\right)=U^{\prime \prime}\left(x_{ \pm}^{0}\right)+U^{(3)}\left(x_{ \pm}^{0}\right) \frac{F}{U^{\prime \prime}\left(x_{+}^{0}\right)}$. The leading contribution to $E_{a}(F)$ and $U^{\prime \prime}\left(x_{ \pm}\right)$leads to an escape rate $k(F)=k_{0} e^{\beta F a}$; this is the celebrated Bell's expression [7]. The basic assumption in the above derivation is that the well and barrier are only slightly shifted away from the original positions. Consequently, Bell's expression is valid when the pulling force is weak.

When $1-F / F_{c} \ll 1, x_{ \pm}$is in the vicinity of $x_{c}$; therefore, the dissociation rate of the bond can be found by expanding $U(x)$ near $x_{c}$. Since $U^{\prime \prime}\left(x_{c}\right)=0$ and $U^{\prime}\left(x_{c}\right)=$ $F_{c}$, one has $U^{\prime}(x)=F_{c}+U^{(3)}\left(x_{c}\right)\left(x-x_{c}\right)^{2} / 2$ at $x$ close to $x_{c}$. If $U^{(3)}\left(x_{c}\right)=0$, then one needs to expand to higher order and $U^{\prime}(x)=F_{c}+U^{(n+1)}\left(x_{c}\right)\left(x-x_{c}\right)^{n} / n$ !. Here $n>$ 2 is even because $U^{\prime}(x)$ has maximum value at $x_{c}$. This corresponds to the following approximation for the poten- tial: $\quad U \cong \frac{F_{c} b}{2}\left[y-y^{n+1} /(n+1)\right]+U\left(x_{c}\right), \quad$ where $y=$ $\left(x-x_{c}\right) /(b / 2)$ and $b=2\left[-n ! F_{c} / U^{(n+1)}\left(x_{c}\right)\right]^{1 / n}$ is the characteristic length of $U$ in this regime. The well and barrier for $V=U-F x$ are therefore located at $x_{ \pm}(F)=$ $\pm \frac{b}{2}\left(1-F / F_{c}\right)^{1 / n}+x_{c}$. The barrier height $E_{a}(F)$ and the intrinsic time scale $\tau(F)$ in this regime are expressed by

$$
\begin{aligned}
E_{a}(F) & =V\left(x_{+}\right)-V\left(x_{-}\right)=E_{a}^{*}\left(1-\frac{F}{F_{c}}\right)^{n+1 / n} \\
\text { and } \quad \frac{1}{\tau(F)} & =\frac{2 \pi \gamma M}{\sqrt{\left|U^{\prime \prime}\left(x_{+}\right) U^{\prime \prime}\left(x_{-}\right)\right|}}=\frac{1}{\tau_{*}}\left(1-\frac{F}{F_{c}}\right)^{n-1 / n},
\end{aligned}
$$

where $E_{a}^{*}=\frac{n}{n+1} b F_{c}$ and $\frac{1}{\tau_{*}}=(n+1) \beta E_{a}^{*} /\left[\pi\left(b^{2} / D\right)\right]$.

Although inserting Eq. (3) into $k(F)=\frac{1}{\tau(F)} \times$ $\exp \left[-\beta E_{a}(F)\right]$ with $\nu=(n+1) / n$ yields an expression similar to the general form in [4], our theory actually is very different from Ref. [4]. The general form in [4] is not derived from the true potential $U(x)$; it is a proposed form with fitting parameters $E_{a}^{0}, a$, and $k_{0}$. Like any reasonable approximation for $U(x)$, this expression reduces to Bell's expression in small $F$ limit. Reference [4] suggests that by fitting the experimental data to their general form a better fit for $E_{a}^{0}, a$, and $k_{0}$, compared to Bell's phenomenological theory, can be obtained, and it does not matter whether the experiment is carried out under $F \ll F_{c}$ or $1-F / F_{c} \ll 1$. However, we show that Bell's expression represents a small perturbation to the thermally activated escape caused by weak pulling. As a result, the intrinsic properties such as $k_{0}$ and $a$ are preserved in Bell's expression but the information about the critical force $F_{c}$ is beyond Bell's expression. On the other hand, when $1-F / F_{c} \ll 1$, the apparent free energy landscape is significantly altered, and thereby $k_{0}$ and $a$ cannot be obtained from the escape rate. In this case the apparent energy barrier is proportional to (1$\left.\frac{F}{F_{c}}\right)^{3 / 2}$. This indicates that one can extract $F_{c}$ from strong pulling experiments. Notice that $F_{c}$ is an important feature of the potential that has been mostly ignored so far. In vivo, the rupture of soft bonds can be thermally assisted or mechanically induced; in the latter case $F_{c}$ is the relevant quantity, not $k_{0}$ or $a$.

To examine our analysis, we consider a model potential

$$
U(x)=\frac{E_{a}^{0}}{2}\left[1-\cos \pi\left(\frac{x}{a}\right)\right], \quad 0 \leq x \leq a .
$$

The escape rate $k$ for this potential under constant force can be obtained analytically. Straightforward algebra leads to $x_{-}=\frac{a}{\pi} \sin ^{-1}\left(\frac{F}{F c}\right), x_{+}=a-x_{-}$, and $F_{c}=\frac{E_{a}^{0}}{2}\left(\frac{\pi}{a}\right)$. The energy barrier and the intrinsic time scale are given by $E_{a}(F)=E_{a}^{0}\left[\sqrt{1-\left(\frac{F}{F_{c}}\right)^{2}}-\frac{F}{F_{c}} \cos ^{-1}\left(\frac{F}{F_{c}}\right)\right]$ and $\frac{1}{\tau(F)}=$ $\tau_{0}^{-1}\left[1-\left(\frac{F}{F_{c}}\right)^{2}\right]^{1 / 2}$, respectively. In the limit $F / F_{c} \ll 1$, one has $E_{a}=E_{a}^{0}\left[1-\frac{\pi}{2} \frac{F}{F_{c}}+\frac{1}{2}\left(\frac{F}{F_{c}}\right)^{2}\right] \approx E_{a}^{0}-F a$ and $\frac{1}{\tau(F)}=$ $\tau_{0}^{-1}\left[1-\frac{1}{2}\left(\frac{F}{F_{c}}\right)^{2}\right] \approx \tau_{0}^{-1}$, consistent with Bell's expression. In the limit $1-F / F_{c} \ll 1$, one has $E_{a}=\frac{2 \sqrt{2}}{3} E_{a}^{0}\left(1-\frac{F}{F_{c}}\right)^{3 / 2}$ 
and $\frac{1}{\tau(F)}=\sqrt{2} \tau_{0}^{-1}\left(1-\frac{F}{F_{c}}\right)^{1 / 2}$, consistent with the generalized Garg form with $n=2$. This confirms that Bell's and Garg's expressions are valid in different regimes.

Constant loading rate and periodic forcing experiments. - Now we consider experiments with constant loading rate, $F(t)=\dot{F}_{t} t$. Although $E_{a}$ and $\tau$ vary with time, the survival probability $P_{s}(t)$ satisfies $\frac{d P_{s}}{d t}=-k(t) P_{s}(t)$ if the Brownian particle is adjusted to the apparent potential, $U(x)-F(t) x, \quad$ instantaneously. Therefore $P_{s}(t)=$ $\exp \left[-\int_{0}^{t} k\left(t^{\prime}\right) d t^{\prime}\right]$, and the mean bond lifetime $\langle T\rangle=$ $\int_{0}^{\infty} t\left(-\frac{d P_{s}}{d t}\right) d t$ is given by

$$
\langle T\rangle=\int_{0}^{\infty} t\left\{k(t) \exp \left[-\int_{0}^{t} k\left(t^{\prime}\right) d t^{\prime}\right]\right\} d t,
$$

and the rupture force $F_{u}=\dot{F}_{t}\langle T\rangle$. Analytical expressions for $\langle T\rangle$ can be obtained for the slow pulling $\dot{F}_{t}\langle T\rangle \ll F_{c}$ and fast pulling $1-\dot{F}_{t}\langle T\rangle / F_{c} \ll 1$ regimes. In the slow pulling regime, the bond lifetime is obtained by substituting Bell's expression $k(F(t))=k_{0} e^{\beta \dot{F}_{t} a t}$ into Eq. (5). The resulting bond lifetime is

$$
\langle T\rangle=\left(\frac{1}{\beta a \dot{F}_{t}}\right) \exp \left(\frac{k_{0}}{\beta a \dot{F}_{t}}\right) E_{1}\left(\frac{k_{0}}{\beta a \dot{F}_{t}}\right) .
$$

Here $E_{1}(x)=\int_{x}^{\infty} \frac{e^{-u} d u}{u}$ is the exponential integral. When $\dot{F}_{t} a / k_{0} \geq k_{B} T$, the change of energy barrier height due to external force during time interval $1 / k_{0}$ is greater than thermal energy. Thus bond lifetime is affected by the pulling, and the above expression can be shown to be reduced to [3] $\dot{F}_{t}\langle T\rangle \approx \frac{1}{\beta a}\left(\ln \frac{\beta a \dot{F}_{t}}{k_{0}}+\lambda\right)$; here $\lambda=0.5772 \ldots$ is the Euler constant. Thus the rupture force grows logarithmically with $\dot{F}_{t}$ in the slow pulling regime. In the fast pulling regime, bond dissociation occurs when the ramped pulling approaches $F_{c}$. Inserting the approximate potential Eq. (3) into Eq. (1), then performing asymptotic integration in Eq. (5) for $\beta E_{a}(F) \gg 1$, yields the generalized Garg form for bond lifetime [8]:

$$
\langle T\rangle \cong \frac{F_{c}}{\dot{F}_{t}}\left\{1-\left[\frac{-\ln \left(\beta b \dot{F}_{t} \tau_{*}\left(\beta E_{a}^{*}\right)^{n-2 / n+1}\right)}{\beta E_{a}^{*}}\right]^{n / n+1}\right\} .
$$

For the most general case $n=2$, rupture force scales as $\left(\ln \dot{F}_{t}\right)^{3 / 2}$. From the above analysis, the much debated question of whether Bell's expression or the generalized Garg form is appropriate to describe constant loading rate experiments [4] becomes clear. One should first chose either Bell's expression or the generalized Garg form to estimate $F_{c}$ from the experimental data, then check whether the experiment is conducted in the weak pulling or strong pulling limit by comparing the rupture force to $F_{c}$. It is not true that one expression is better than another in all experiments, but it is possible that many existing experimental data are actually taken from experiments conducted in the strong pulling limit [4].

Experiments on the dissociation of a single bond under time-periodic force $F(t)=F_{0} \cos \left(2 \pi t / \tau_{p}+\alpha\right)$ can also be done. When $\langle T\rangle \gg \tau_{p}$, in the small amplitude regime $F_{0} / F_{c} \ll 1$ Bell's expression is valid, and by substituting $F(t)$ into Bell's expression one finds

$$
\langle T\rangle \approx\left[k_{0} I_{0}\left(\beta F_{0} a\right)\right]^{-1},
$$

for $\beta F_{0} a \leq 4$. In the large amplitude regime $1-$ $F_{0} / F_{c} \ll 1$, the lifetime is obtained by applying the generalized Garg form Eq. (3):

$$
\langle T\rangle \approx \frac{1}{k\left(F_{0}\right)} \sqrt{2 \pi \frac{n+1}{n} \beta E_{a}^{*}\left(1-\frac{F_{0}}{F_{c}}\right)^{1 / n} \frac{F_{0}}{F_{c}},}
$$

where $k\left(F_{0}\right)=\frac{1}{\tau\left(F_{0}\right)} \exp \left[-\beta E_{a}\left(F_{0}\right)\right]$ denotes the rate constant associated with constant pulling $F_{0}$. Therefore in both limits $\langle T\rangle$ increases with $F_{0}$ but is independent of $\tau_{p}$. The lifetime is independent of the frequency of the force for the following reason. In each period, the escape probability is
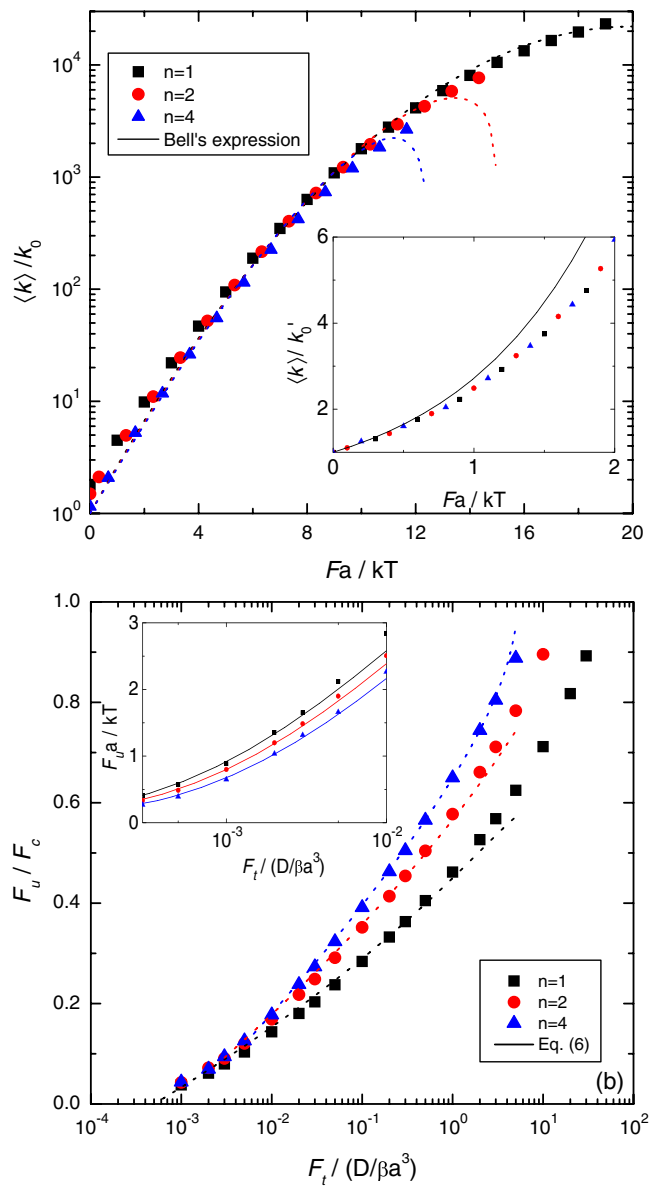

FIG. 1 (color online). Comparisons between asymptotic expressions and Langevin dynamics simulations for $U^{\prime}(x)=\{1-$ $\left.\left[\left|x-x_{c}\right| /(b / 2)\right]^{n}\right\}(n+1) E_{a}^{0} / n b$ with $n=1,2$, and 4 . The activation energy for different potentials is the same, $\beta E_{a}^{0}=10$. (a) Constant-force escape for $\langle k\rangle=\langle T\rangle^{-1}$ and (b) constant loading rate escape for $F_{u} / F_{c}$. The dashed lines depict the expressions based on the generalized Garg form [Eqs. (3) and (7)]. The insets show (a) the weak force regime and (b) the slow pulling regime, where Bell's expression [Eqs. (2) and (6)] is valid. The intrinsic rate constant $k_{0}^{\prime}$ is determined by simulations. 


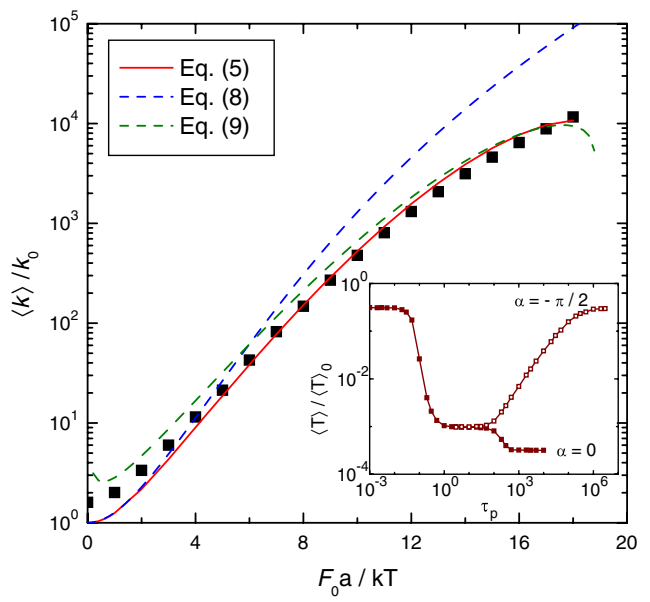

FIG. 2 (color online). Bond lifetime under time-periodic force $F(t)=F_{0} \cos \left(2 \pi t / \tau_{p}+\alpha\right)$ in the $\tau_{p}$-independent regime. $\langle T\rangle_{0}$ denotes the intrinsic bond lifetime for $U(x)=6[1-$ $\cos (\pi x / a)]$. The lines represent numerical solution of Kramers rate theory [Eq. (5)] and asymptotic expressions [Eqs. (8) and (9). Since we have adopted a periodic $U(x)$ for simulations, $\langle T\rangle_{0}=k_{0}^{-1}$ evaluated by Eq. (1) is about twice greater than that obtained from simulations.

largest in the vicinity of $F_{0}$, and this escape window is $\sim \tau_{p}$. Since the occurring frequency of this window is proportional to $\tau_{p}^{-1}$, the total escape window over many periods is independent of $\tau_{p}$ as long as $\langle T\rangle \gg \tau_{p}$.

Discussion. - To illustrate the ideas presented in our previous discussion, consider potentials with $U^{\prime}(x)=\{1-$ $\left.\left[\left|x-x_{c}\right| /(b / 2)\right]^{n}\right\}(n+1) E_{a}^{0} / n b$ for $n=1,2$, and 4 . The intrinsic barrier height is $E_{a}^{0}$, the critical force for given $n$ is $F_{c}=(n+1) E_{a}^{0} / n b$, and different $n$ corresponds to different potential shape. Langevin dynamics simulations are performed for $\beta E_{a}^{0}=10$ to get $k$ for constant force [Fig. 1(a)] and $F_{u}$ for constant loading rate [Fig. 1(b)] escape. In Fig. 1, simulation results are compared to results from Bell's and Garg's expressions. The insets demonstrate the validity of Bell's expression in weak (constant force) and slow (constant loading rate) pulling regimes; both Figs. 1(a) and 1(b) show that the generalized Garg forms agree with the simulation results over a wide range of external forces and loading rates. From Fig. 1(a) we also find that it is difficult to distinguish constant-force simulation results for different choices of $n$ with the same $\beta E_{a}^{0}=10$, except when the external force is very close to or exceeds the $F_{c}$ of some chosen $n$. This suggests that bond lifetime in most constant-force experiment is dominated by the intrinsic barrier $E_{a}^{0}$, not by the details of the potential surface (in this case, $n$ or $F_{c}$ ). On the other hand, distinct $F_{u}$ results are obtained in Fig. 1(b) for different choices of $n$. Under the same loading rate, the rupture force increases with $n$. Thus the constant-force pulling experiment is more useful in extracting $E_{a}^{0}$ than the constant loading rate one, while it has been pointed out $[3,9]$ that the latter is suitable to probe the details of the free energy landscape close to $F_{c}$.
Langevin dynamics simulations are also performed for periodic forcing for the potential given by Eq. (4). As shown in the inset of Fig. 2, at very small $\tau_{p}$ the adiabatic approximation is not valid and the lifetime approaches a constant value independent of $F_{0}$, which is not of practical experimental interest. At very large $\tau_{p}(\gg\langle T\rangle)$, the bond lifetime approaches another constant for $\alpha=0$ because the bond is effectively under constant force $F_{0} \cos \alpha$ when it breaks. For $\alpha=-\pi / 2$, the bond lifetime at very large $\tau_{p}$ becomes the constant loading rate lifetime with $\dot{F}_{t}=$ $2 \pi F_{0} / \tau_{p}$. In the intermediate $\tau_{p}$ where the adiabatic approximation is valid and still $\tau_{p} \gg\langle T\rangle$, there is indeed a region where $\langle T\rangle$ is independent of $\tau_{p}$ and Eq. (8) or (9) can apply, depending on the magnitude of $F_{0}$. Figure 2 shows $\langle k\rangle=\langle T\rangle^{-1}$ as a function of $F_{0}$ for $\tau_{p}$-independent region simulations. Equation (9), based on a general Garg form, describes $\langle k\rangle$ with high precision at large $F_{0}$, while Eq. (8), based on Bell's expression, describes $\langle k\rangle$ well at small $F_{0}$ (data not shown). This consequence indicates that periodic forcing experiments allow one to extract $k_{0}$ and $a$ at small $F_{0}$, and $F_{c}$ and $E_{a}^{*}$ at large $F_{0}$.

In summary, we have derived both Bell's expression and the generalized Garg form for forced escaped problem; they are approximations to describe single-molecule pulling experiments in different regimes. Our study suggests that the constant-force experiment is useful for extracting the intrinsic barrier height of the potential, the constant loading rate experiment is useful to study the shape of free energy landscape close to the inflection point, and the periodic forcing experiment can be used to find the intrinsic dissociation rate and the value of critical force. Therefore new ways to probe the inner lives of biomolecular complexes are revealed, and they should be easily checked in future experiments.

This work is supported by the National Science Council of Taiwan.

*Corresponding author.

[1] E. L. Florin, V. T. Moy, and H. E. Gaub, Science 264, 415 (1994).

[2] R. Merkel, P. Nassoy, A. Leung, K. Ritchie, and E. Evans, Nature (London) 397, 50 (1999); Y.-S. Lo, Y.-J. Zhu, and T. P. Beebe, Jr., Langmuir 17, 3741 (2001).

[3] Y.-J. Sheng, S. Jiang, and H.-K. Tsao, J. Chem. Phys. 123, 091102 (2005).

[4] O. Dudko, G. Hummer, and A. Szabo, Phys. Rev. Lett. 96, 108101 (2006).

[5] H. A. Kramers, Physica (Amsterdam) 7, 284 (1940).

[6] P. Hänggi, P. Talkner, and M. Borkovec, Rev. Mod. Phys. 62, 251 (1990).

[7] G. I. Bell, Science 200, 618 (1978).

[8] A. Garg, Phys. Rev. B 51, 15592 (1995).

[9] H.-Y. Chen and Y.-P. Chu, Phys. Rev. E 71, 010901(R) (2005). 